\title{
Azelastine hydrochloride, a dual-acting anti-inflammatory ophthalmic solution, for treatment of allergic conjunctivitis
}

This article was published in the following Dove Press journal:

Clinical Ophthalmology

30 August 2010

Number of times this article has been viewed

\author{
Patricia B Williams ${ }^{1,2}$ \\ Elizabeth Crandall ${ }^{2}$ \\ John D Sheppard ${ }^{1,2}$
}

'Thomas R Lee Center for Ocular Pharmacology, ${ }^{2}$ Department of Ophthalmology, Eastern Virginia Medical School, Norfolk, VA, USA
Correspondence: Patricia B Williams

Thomas R Lee Center for Ocular Pharmacology, Eastern Virginia Medical School, PO Box 1980, Norfolk, VA 23507, USA

Tel +I 7574465630

Fax +I 7576242270

Email williap@evms.edu

\begin{abstract}
Over 50\% of patients who seek treatment for allergies present with ocular symptoms. Our current ability to control ocular allergic symptoms is greater than ever before. Newer dualacting topical eyedrops attack multiple facets of the allergic cascade. Azelastine has antihistaminic effects providing immediate relief, mast cell stabilization providing early-phase intervention, and inhibition of expression and activation of anti-inflammatory mediators which characterize the late phase of the immune reaction. The ophthalmic eyedrop formulation is approved for treatment of allergic conjunctivitis in adults and children aged over 3 years. In clinical trials comparing azelastine with other dual-acting eyedrops, such as levocabastine and olopatadine, azelastine was reported to be slightly less efficacious and to sting briefly upon administration. Even so, many patients experienced the full benefit of symptom relief, and preferred azelastine. As a broad-spectrum drug, azelastine offers many desirable properties for management of ocular allergies. Because it can often produce maximal effect with just twice-daily dosing, azelastine is a particularly good choice for the allergic population in whom minimizing exposure to topical products and preservatives is a key concern.
\end{abstract}

Keywords: allergic conjunctivitis, dual acting anti-inflammatory, $\mathrm{H}_{1}$ receptor antagonism, mast cell stabilization, inflammatory mediator inhibition

\section{Introduction}

More than 50 million Americans seek treatment for allergies annually, and over half of these patients present with ocular symptoms. ${ }^{1}$ Allergy commonly attacks the conjunctival mucosa, as well as the nose, sinuses, ears, upper airways, and lungs. Remarkably, the ocular component may be the most common and initially the most prominent life-altering symptomatic complaint. Seasonal or episodic allergy patients become ill for only a few weeks or days, while others have symptoms persisting throughout the year or their entire lifetime.

The associated health care costs related to allergic conjunctivitis are often comingled with allergic rhinitis, and have been reported to be as high as \$6 billion in the US, with $25 \%$ of that amount due to medication costs. ${ }^{2,3}$ Fiscal outlays attributed to ocular prescription medication have risen rapidly in the past generation, from $\$ 6$ million in 1990 to \$200 million recently, with projected sustained growth of over $20 \%$ per year. Remarkably, this prescription growth is unique in that it is not commensurate with the aging of the population, but is concomitant with population growth. The prescription growth is due in part to vastly improved medication efficacy and its obvious superiority over less specific, less potent, and more toxic over-the-counter products. The actual cost of the medications and their relative price increases have continued recently on 
a yearly basis, while a wide variety of formerly proprietary allergy medications have gone generic, or over-the-counter, with blossoming direct-to-consumer campaigns. With these numbers in mind, it is clear that cost-effective care for ocular, cutaneous, and respiratory allergy is a valuable asset.

Allergic conjunctivitis has several clinical hallmarks. Most patients experience acute attacks of red, irritated eyes with an intense feeling of itching that result in tearing. These episodes are often accompanied by clinical signs of lid edema, conjunctival chemosis, and papillary reactions that can be appreciated on examination. The underlying pathophysiology originates from the body's natural immune reactions. Conjunctival tissue harbors numerous mast cells that are filled with histamine and other inflammatory components. Due to the priming mechanisms of mast cells, previous sensitivities to specific allergens cause antibodies to form against these allergens. These antibodies are highly integrated into the cell surface of the mast cells. Then, upon re-exposure of the tissue to the allergen, the surface antibodies on mast cells initiate a cascade of events that results in the release of their granular contents. These agents are the inciting factors for the classic symptoms of allergic conjunctivitis. The early phase of the allergic reaction is due to histamine-induced vasodilation that results in redness and fluid collection in the form of lid edema and chemosis, and also causes the classic symptom of pruritus. The release of other inflammatory mediators from mast cells recruits additional mediators to the site, consequently sustaining the late phase of the allergic reaction. Thus, the symptomatology of allergic conjunctivitis centers around the effects of histamine and inflammatory mediators on the surrounding ocular tissues. Given the multitude of mediators involved, drugs like azelastine (Optivar ${ }^{\circledR}$; Bausch and Lomb, Rochester, NY) that counteract several steps along this pathway can provide clinically efficacious treatment of patients with allergic conjunctivitis.

For ocular allergies, the latest generation of topical antiallergic medications possesses multiple actions that include antihistaminic effects to provide immediate relief, earlyphase intervention through stabilization of activated mast cells, and additional late-phase reactant inhibition without the customary topical or systemic steroid side effect profiles. ${ }^{4}$ Azelastine, a member of this newest generation, has multiple effects as an antihistamine, mast cell stabilizer, and inhibitor of inflammatory mediators. Azelastine was first approved in 1996 as a nasal spray to treat symptoms of seasonal allergic disease and in 1999 for nonallergic vasomotor rhinitis. An ophthalmic eyedrop formulation for the treatment of allergic conjunctivitis in adults and children over three years of age received Food and Drug Administration approval in May $2000 .^{5}$

\section{Pharmacology of azelastine Chemical classification}

Azelastine is a selective, nonsedating $\mathrm{H}_{1}$ antagonist with structural similarities to other antihistamines. As a phthalazinone derivative with a seven-membered ring, it is commonly used as an anhydrous monohydrochloride salt (Figure 1). The white, odorless, bitter-tasting crystalline powder is a racemic mixture of azelastine hydrochloride, with a high melting point of $225^{\circ} \mathrm{C}$. Even as a hydrochloride salt, the small organic molecule is only sparingly soluble in methanol, propylene glycol, and water, but slightly soluble in glycerin, ethanol, and octanol. Although azelastine is soluble in dichloromethane and in chloroform, these solvents are not well tolerated in pharmaceutical preparations.

\section{Dosage form}

Although azelastine is orally active, it is most often administered topically, either as a nasal spray or as a sterile ophthalmic solution. The ophthalmic solution is not lightsensitive and is stable for up to two years when packaged in an unopened dropper bottle and stored upright at $35-75^{\circ} \mathrm{F}$. Once opened, the unused contents should be discarded after one month.

Azelastine ophthalmic solution ( $0.05 \%)$ is preserved with benzalkonium chloride (BAK). The toxic effects of BAK on the cornea are well documented. ${ }^{6}$ In the concentration used here (BAK 0.125\%), prolonged use of topical eyedrops containing BAK is associated with well documented deleterious effects on the conjunctiva and cornea, as well as the quality and quantity of tear film. Because BAK may be absorbed by soft contact lenses, patients should not insert contact lenses for at least 10 minutes after instilling azelastine eyedrops. In addition to BAK, the aqueous vehicle is composed of ingredients commonly used in generally well tolerated eyedrops. The vehicle includes disodium

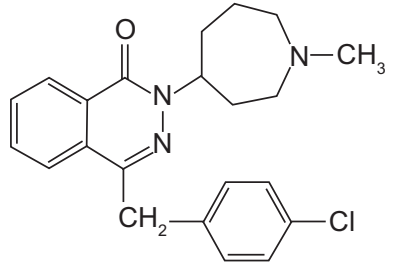

- $\mathrm{HCl}$

Azelastine

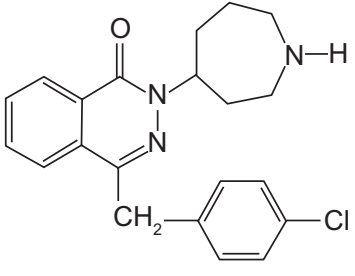

Desmethylazelastine
Figure I Structure of azelastine and its major metabolite. 
ethylenediaminetetraacetic acid, hypromellose, sorbitol solution, and sodium hydroxide buffered to $\mathrm{pH}$ 5.0-6.5; osmolarity is about $300 \mathrm{mOsmol} / \mathrm{L}$.

About one third of patients remark that azelastine eye drops sting transiently upon instillation. This irritating effect may be due to the low $\mathrm{pH}$ or, more probably, due to intrinsic irritating effects of azelastine on the ocular epithelium. ${ }^{4}$ Chemical properties of azelastine may directly cause irritation of ocular tissues after topical administration. Conjunctival cell damage by topical antiallergic drugs can be measured in cultured rabbit conjunctiva using the lactate dehydrogenase assay. In comparison with olopatadine (Patanol ${ }^{\circledR}$; Alcon, Fort Worth, TX), both azelastine and ketotifen increased lactate dehydrogenase titers, edema, and cell degeneration, which is reflected in the clinical data discussed in this review. ${ }^{7}$

\section{Multiple mechanisms of action}

Azelastine is classified pharmacologically as a secondgeneration antihistamine, ie, it is a relatively selective, nonsedating, competitive antagonist at $\mathrm{H}_{1}$ receptors. Its relative lack of central nervous system activity distinguishes it from first-generation antihistamines. Inhibition of inflammatory mediators, in addition to antihistaminic and mast cell stabilizing effects, place it among the new generation of dualacting anti-inflammatory drugs. In addition to azelastine's high affinity for $\mathrm{H}_{1}$ receptors, its ability to modify several other mediators of inflammation and allergy contributes to its mechanism of action. ${ }^{8}$ In vitro and in vivo studies, as well as the clinical trials discussed here, support the dual effects of direct inhibition and stabilization of inflammatory cells (Figure 2).

In vitro data indicate that azelastine's affinity for $\mathrm{H}_{1}$ receptors is estimated to be several times greater than that of chlorpheniramine, a first-generation $\mathrm{H}_{1}$ antagonist. ${ }^{9}$ Azelastine has only weak affinity for $\mathrm{H}_{2}$ receptors. Release of histamine from mast cells is also inhibited possibly by reversible inhibition of voltage-dependent L-type calcium channels. ${ }^{10}$ Inhibition of mast cell degranulation may also decrease the release of other inflammatory mediators, including leukotrienes and interleukin-1 $\beta$, among others. Azelastine also directly antagonizes other mediators of inflammation, such as tumor necrosis factor- $\alpha$, leukotrienes, endothelin-1, and platelet-activating factor. ${ }^{11,12}$

Conjunctival mast cells play a prominent role in allergic conjunctivitis. Due to its multiple mechanisms, azelastine mediates both immediate- and late-phase allergic reactions associated with mast cell degranulation. ${ }^{13}$ By directly antagonizing the effects of histamine released from mast cells, azelastine modifies immediate allergic responses typified by pruritus, hyperemia, edema, and chemosis. Activation of $\mathrm{H}_{1}$ receptors on nerve tissue causes pruritus, while activation of $\mathrm{H}_{1}$ receptors on vascular tissue causes hyperemia and

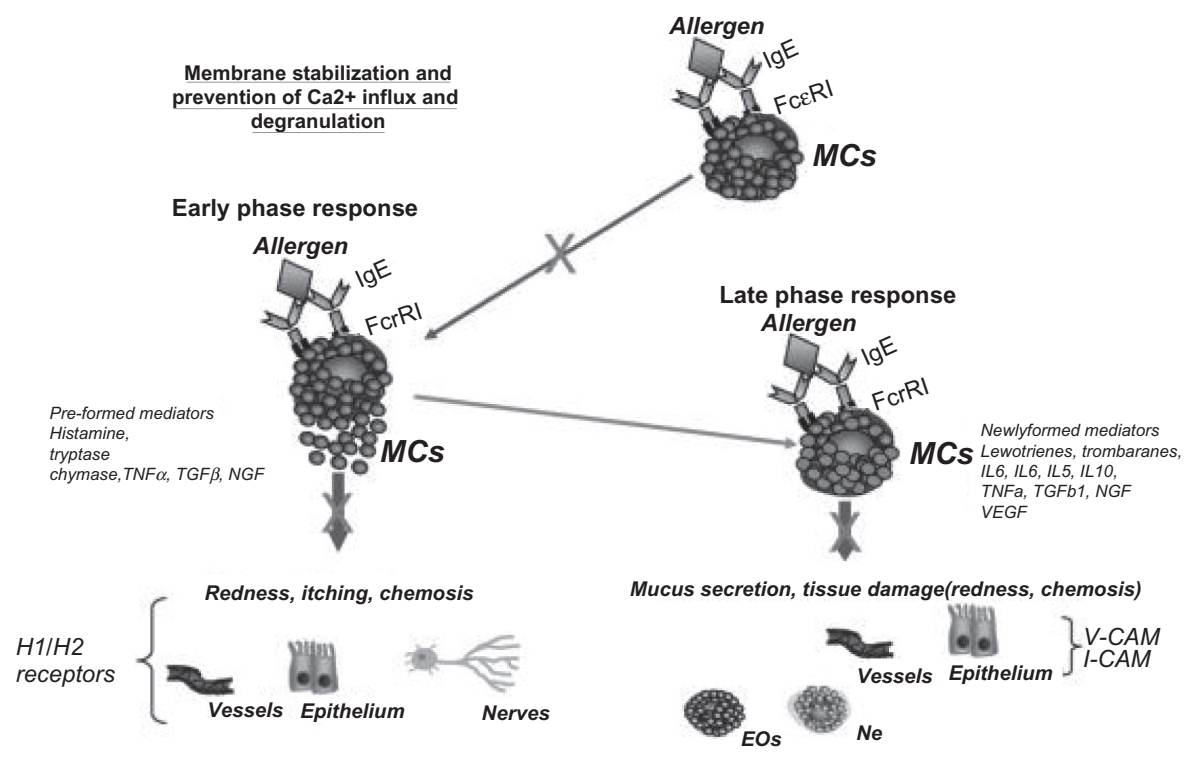

Figure 2 Multiple pathways are responsible for the antiallergic effects of azelastine.

The figure summarizes the pathways of action of multiple action antiallergic drugs, which include membrane activation and cell degranulation (early phase reaction) and de-novo synthesis (late-phase reaction). Drugs acting at the membrane level also interfere with the subsequent steps of intracellular $\mathrm{Ca}^{2+}$ influx and PTK signal transduction pathway. Copyright (C) 2009, Lippincott Williams \& Wilkins. All rights reserved. Adapted with permission from Lambiase A, Micera A, Bonini S. Multiple action agents and the eye: do they really stabilize mast cells? Curr Opin Allergy Clin Immunol. 2009;9(5):454-465.

Abbreviations: IgE, immunoglobulin E; MC, mast cell; NGF, nerve growth factor; PTK, protein-tirosyne kinase; VEGF, vascular endothelial growth factor. 
edema. Through both IgE-dependent and IgE-independent inhibition of mast cell degranulation, azelastine diminishes the second wave of newly generated biochemical mediators that increase mucus secretion and promote infiltration of inflammatory cells. Azelastine minimizes ocular surface damage through inhibition of immune cells that characterize the late-phase immune reaction, including downregulation of intercellular adhesion molecule (ICAM)-1 on conjunctival epithelial cells. ${ }^{14}$

In vivo studies in a guinea pig model showed that both histamine-related and histamine-independent bronchoconstriction were inhibited by azelastine. ${ }^{8}$ Furthermore, vascular extravasation was reduced by azelastine in sensitized guinea pig and rat models. Both immediate- and late-phase responses were inhibited by azelastine in a mouse model sensitized by dintrofluorobenzene. Azelastine retains its efficacy over a sustained period of time. Regular daily use has not been associated with tachyphylaxis due to downregulation of $\mathrm{H}_{1}$ receptors or their antagonism. ${ }^{15}$

\section{Pharmacokinetics}

\section{Absorption and distribution}

Azelastine is well absorbed after oral administration. ${ }^{16}$ Plasma concentrations were linear over the dosage range $(2.2-17.6 \mathrm{mg})$. Both the time to maximum plasma concentration ( $\mathrm{t}_{\max } 4.6$ hours) and elimination half-life $\left(t_{1 / 2} 25.0 \pm 5.2\right.$ hours) were independent of dose. ${ }^{17,16}$ The large interindividual variation is likely due to differences in enterohepatic circulation of this lipid-soluble drug. Similarly, following intravenous administration, the $t_{1 / 2}$ was 22 hours, volume of distribution at steady state was $14.5 \mathrm{~L} / \mathrm{kg}$, and plasma clearance was $0.5 \mathrm{~L} / \mathrm{h} / \mathrm{kg}$ (see http://dailymed.nlm.nih.gov). Plasma protein binding ( $88 \%$ of unmetabolized drug) is extensive.

By comparison, following ocular administration, systemic absorption of azelastine is minimal. After 56 days of topical treatment two to four times daily, plasma concentrations were only $0.2-0.25 \mathrm{ng} / \mathrm{mL}$. These low plasma concentrations decrease the probability of systemic adverse effects, and also imply that the response to azelastine eyedrops is due to a local effect on ocular tissue. Using an allergen challenge design, ${ }^{18}$ azelastine had an onset of action within three minutes, and the effects lasted at least 8-10 hours.

\section{Metabolism and elimination}

After oral administration, N-desmethylazelastine, the primary active metabolite of azelastine, is produced in the liver largely by CYP450, CYP3A4, and CYP2D6 ${ }^{19}$ (Figure 1). Lesser amounts of two inactive carboxylic acid metabolites are also produced. ${ }^{20}$ The $t_{1 / 2}$ of $\mathrm{N}$-desmethylazelastine (54 hours) is more than twice as long as azelastine, and contributes greatly to its long duration of action after systemic administration. Severe adverse effects have been reported for antihistamines that are metabolized by the liver, which provides a cogent argument for topical administration. Topical azelastine eyedrops, administered to the target organ, permit application of relatively small doses. About $75 \%$ of azelastine is excreted in the feces, and more than $90 \%$ is in the form of N-desmethylazelastine, its primary metabolite. ${ }^{21}$

Less than $25 \%$ of azelastine and its metabolite is excreted in the urine, but in patients with compromised renal function (ie, $\mathrm{C}_{\mathrm{cr}}<50 \mathrm{~mL} / \mathrm{min}$ ), the plasma concentrations can increase by up to $75 \%$. The implication of declining renal function is noted in patients older than 65 years, in whom oral administration increased the $\mathrm{t}_{1 / 2}$ to $38 \pm 15.3$ hours, with a concomitant doubling of the $\mathrm{C}_{\max } \cdot{ }^{17}$ Given the long $\mathrm{t}_{1 / 2}$ of N-desmethylazelastine, it too would be expected to accumulate and further extend the duration of action, particularly during multiple dosing, as is common in the treatment of allergic conjunctivitis. Despite the potential for increased plasma concentration and duration of action, azelastine is well tolerated in older patients. Given that systemic absorption of azelastine after topical administration is minimal, most elderly patients tolerate this form of administration very well. Likewise, in placebo-controlled studies, both topical ocular and nasal administration of azelastine are well tolerated in children aged $5-12$ years..$^{22,23}$

\section{Drug interactions}

Concomitant administration of more than one medication can precipitate severe adverse effects. Many clinically relevant drug interactions are due to unexpectedly increased plasma concentrations of one or both drugs, when both are metabolized by the same CYP450 enzymes. Of particular concern are the arrhythmogenic changes in the QT interval when an antihistamine, such as terfenadine, is administered orally along with either ketoconazole or erythromycin. ${ }^{24}$ Even though azelastine is more than $90 \%$ metabolized by CYP3A4 and CYP2D6, and to a lesser extent by CYP1A2, ${ }^{19}$ such drug interactions are unlikely to be due to the low plasma concentration of azelastine and N-desmethylazelastine after topical administration.

\section{Carcinogenesis, mutagenesis, and fertility}

Oral doses in mice have demonstrated carcinogenicity, mutagenicity, and fetotoxicity in mice at doses of $68.6 \mathrm{mg} / \mathrm{kg}$, which is 57,000 times greater than the recommended dose for 
topical eyedrops in humans (see http://dailymed.nlm.nih.gov). The pregnancy category is C. There are no well controlled studies of topical application in pregnant women. Likewise, the extent of azelastine excretion in human breast milk is unknown. Considering that other lipophilic drugs are excreted in breast milk, it is likely that azelastine and its active metabolite are too. Given the lack of definitive data, azelastine should be used with great caution in pregnant or nursing women only if the benefit is deemed to be far greater than the potential risk to the progeny.

\section{Efficacy and tolerability studies}

An early study demonstrated that azelastine not only has a rapid onset of action, but also has a protective effect when administered prior to allergen exposure. ${ }^{25}$ This double-masked, placebocontrolled trial included 20 subjects with known Parietaria judaica sensitivity, which was confirmed with a skin prick test to determine the concentration needed to elicit a conjunctival response reproducibly. In the first arm of the study, the allergen was instilled into both eyes. The response was scored every 5 minutes for 20 minutes before and for 30 minutes after instillation of either azelastine or placebo. The score was based on clinical evaluation of conjunctival hyperemia, itching, lacrimation, and eyelid swelling. Compared with placebo, azelastine significantly reduced the symptoms within 10-20 minutes. ${ }^{25}$ The prophylactic effect of azelastine was demonstrated by comparing the response to the allergen challenge after seven days of twice-daily administration of azelastine with placebo. There were no reported adverse effects and the azelastine drops were well tolerated. The scores were recorded 5, 10, 20, and 30 minutes (early-phase reaction), and 6 hours (late-phase reaction) after allergen exposure. As with the first experiment, compared with placebo, azelastine significantly reduced symptom scores during both the early- and late-phase reactions. In addition, impression cytology demonstrated that azelastine significantly decreased both inflammatory cell infiltration and ICAM-1 expression during both the early- and late-phase reactions. Both subjective (symptom scores) and objective (impression cytology) parameters established the antiallergic activity of azelastine in the eye, and confirmed its inhibitory effect on infiltration of inflammation and downregulation of ICAM-1, which was first observed in the nasal mucosa. ${ }^{14}$

This initial trial supported further investigation into the efficacy and safety profiles of azelastine. In a dose-ranging trial, 151 patients with at least one year of allergic conjunctivitis symptoms were randomized to three groups receiving either placebo, or $0.025 \%$ or $0.05 \%$ azelastine twice daily for 14 days. ${ }^{26}$ After seven and 14 days of treatment, patients' symptoms were scored by the investigators on a four-point scale for presence and intensity of itching, lacrimation, and conjunctival erythema. Much like the previous study, azelastine effectively decreased symptoms of allergic conjunctivitis. Comparing the two strengths, $0.05 \%$ azelastine was significantly more effective than $0.025 \%$ azelastine ( $82 \%$ responders versus $73 \%$, respectively). No serious adverse events were reported in any of the three treatment

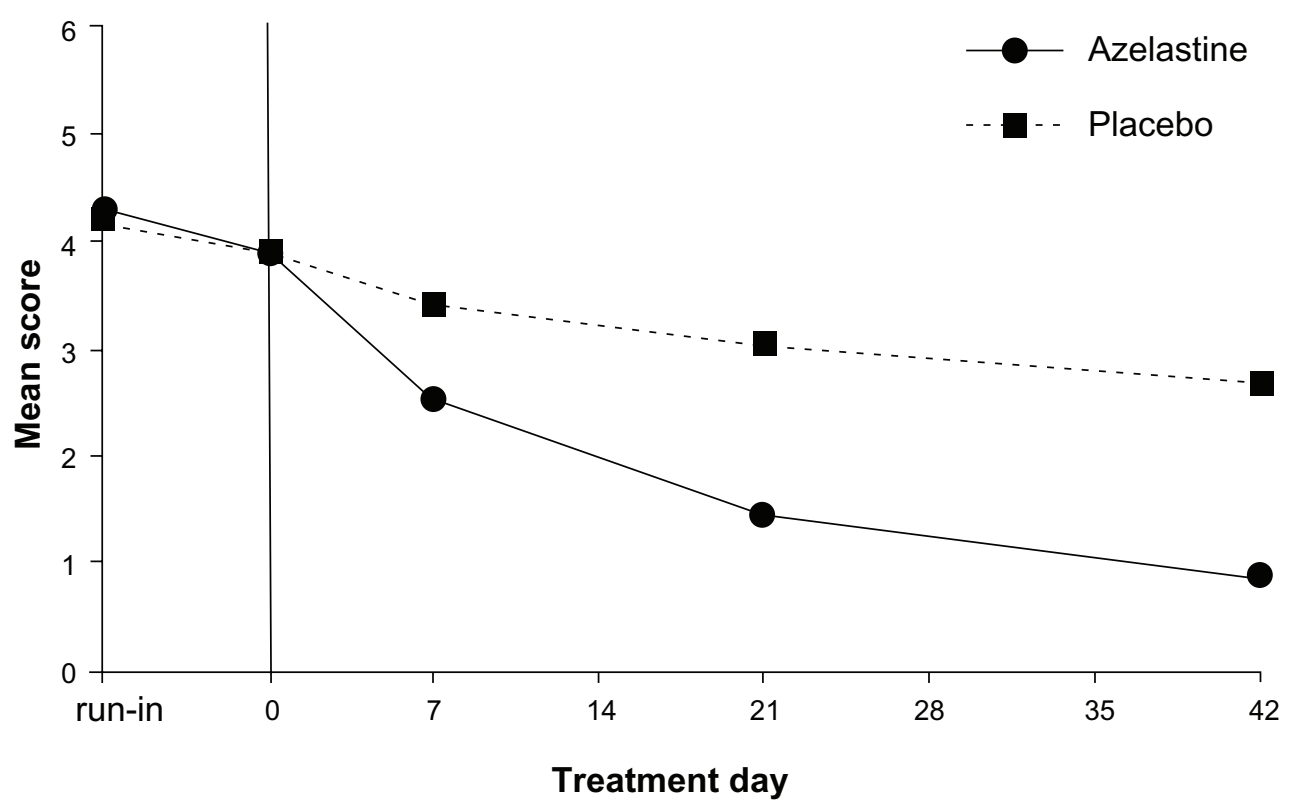

Figure 3 Azelastine topical eye drops cause a sustained decrease in allergic symptoms. Copyright (C) 2003. Reproduced with permission from Nazarov O, Petzold U, Haase H, Nguyen DT, Ellers-Lenz B, Hermann R. Azelastine eye drops in the treatment of perennial allergic conjunctivitis. Arzneimittelforschung. 2003;53:167-I73.27 
groups. However, in contrast with the initial trial, the incidence of treatment-related adverse effects in the azelastine treatment groups was $30.5 \%$. The two most frequently reported adverse effects were burning sensation in the eyes after drug application and a bitter taste. Taste perversion is thought to be the result of the medication draining from the lacrimal system through the nares and then onto the posterior surface of the tongue.

After the efficacy of azelastine was established, further studies investigated the onset and duration of effectiveness of azelastine in patients with allergic conjunctivitis. ${ }^{18}$ Patients with at least a 2-year history of allergic conjunctivitis, a positive allergen skin test, and two separate positive responses to a conjunctival allergen were enrolled in a randomized, blinded clinical trial $(n=80)$. Twenty minutes prior to exposure to the known conjunctival allergen, one eye was treated with placebo and the contralateral eye with azelastine $0.05 \%$. Using a five-point scale, the investigators rated the extent of chemosis and conjunctival redness. The patients also assessed the extent of itching and tearing using the same scale. After a 7-day washout period, the test was repeated, except that the drops were administered 8-10 hours before exposure to the allergen. The results from both experiments confirmed that azelastine was highly effective in decreasing allergic conjunctivitis symptoms, including itching, conjunctival redness, tearing, and chemosis. Onset of action was rapid, occurring within 3 minutes of allergen exposure. Azelastine inhibited development of symptoms for at least 8-10 hours after instillation, suggesting a long duration of action and supporting twice daily administration.

A third double-masked, randomized, placebo-controlled study evaluated the efficacy of azelastine during extended treatment. ${ }^{27}$ Patients were randomized to either placebo or azelastine $(0.05 \%)$ treatment groups $(\mathrm{n}=16 \mathrm{each})$ and followed for 42 days. Following 7, 21, and 42 days of treatment, investigators examined the patients and rated the extent of conjunctival redness and itchy sensation on a scale of $0-6$. The sum score of symptoms showed significant rapid improvement in the azelastine group compared with the placebo group, with continued improvement even up to day 42 (Figure 3). ${ }^{27}$ After 7 days, $55 \%$ in the azelastine group had significant score improvement versus $15 \%$ in the placebo group. After 42 days, the response rate increased to $95 \%$ for azelastine versus $33 \%$ for placebo. The adverse effect profile reported by patients was similar to that reported in other trials. The two most common complaints were taste perversion and a mild stinging or burning sensation after drop administration. In this trial, a slightly larger proportion of patients (22\%) commented on a bitter taste after azelastine. However, patients also reported $97 \%$ tolerability. The very high level of tolerability suggests that the relief of symptoms far outweighs any taste perversion. While confirming that azelastine effectively decreases symptoms of treatment for allergic conjunctivitis, this trial established that azelastine also provides sustained benefit and is well tolerated over time.

Children comprise a large portion of the patient population presenting with allergic symptoms. Once safety and efficacy were established in adults, a separate trial was conducted in children. ${ }^{22} \mathrm{~A}$ double-masked, multicenter study compared the effectiveness of azelastine $(0.05 \%)$ on seasonal conjunctivitis and rhinoconjunctivitis, with that of placebo or levocabastine $0.05 \%$. The children, aged $4-12$ years, were randomized into three treatment groups $(\mathrm{n}=113 \mathrm{each})$. All drops were administered bilaterally twice daily in the morning and evening for 14 days. On days 3 and 14, the investigators evaluated the effectiveness of the medication by scoring three main symptoms, ie, eye itching, lacrimation, and conjunctival redness, on a four-point scale. Patients also kept a journal to document their daily symptoms using the same scale. By comparing mean scores for the three treatment groups, it was clear that the two drug treatments were superior to placebo from both the clinicians' and patients' perspectives. In the investigatorcollected data, treatment was considered satisfactory in 39\% of the placebo group, $74 \%$ of the azelastine group, and $84 \%$ of the levocabastine group. According to the subjects' scores, the azelastine (72\%) and levocabastine (71\%) treatments were far superior to the placebo treatment (41\%). No statistically significant difference was found between the azelastine and levocabastine groups; both were far superior in treatment response than placebo. The adverse effect profile for azelastine reflected that reported in trials with adult patients. The most common adverse effect was a burning or tingling sensation after application of the drops in $33 \%-34 \%$ of the azelastine group, $38 \%$ of the levocabastine group, and $17 \%$ of the placebo group. A bitter taste was reported by two patients. This study confirmed that the safety and efficacy of azelastine in children was similar to that reported in adult patients.

\section{Comparison with other topical antihistamines}

After the safety, efficacy, and tolerability of azelastine were established, other trials were conducted to compare its effectiveness and tolerability with that of other similar drugs. A single-masked, multicenter study compared azelastine $0.05 \%$ with another topical antihistamine, levocabastine, and placebo. ${ }^{28}$ Patients were divided into three treatment 
groups and treated twice daily for 6 weeks $(n=139)$. Before beginning the six-week trial, all subjects underwent a 1-week trial with placebo. Only those subjects who scored $\geq 3$ or on a 0-6-point scale for conjunctival redness and itching were then randomized to one of the three treatment groups. The subjects were evaluated on days 7, 21, and 42 of treatment using the same scale. The treatment response was evaluated by comparing change in symptom score after each treatment evaluation with the initial 1-week placebo score. ${ }^{28}$ Azelastine and levocabastine decreased conjunctival redness and itching by a similar amount, but both were significantly more efficacious than placebo. Because both medications produced very similar responses, the authors concluded that azelastine was at least as effective as levocabastine in treating allergic conjunctivitis.

\section{Adverse effects}

When compared with levocabastine over a six-week period, adverse effects reported by the azelastine treatment group were application site reaction $(26.3 \%)$, taste perversion (10.5\%), and conjunctivitis (1.8\%). ${ }^{28}$ By comparison, $15.4 \%$ of levocabastine users reported application site reaction and no taste changes or conjunctivitis, and only $5.4 \%$ of placebo users reported an application site reaction. Thus, the study results yielded two main conclusions, ie, that azelastine and levocabastine both significantly improve patients' symptoms with comparable efficacy, and that the incidence of adverse effects is greater during treatment with azelastine.

Because olopatadine is another major drug in this class, comparison of azelastine and olopatadine is essential for fully understanding azelastine's place among the therapeutic options. Both are highly efficacious, although their effects may differ somewhat at a cellular basis. ${ }^{5}$ There are several studies comparing their clinical effectiveness. Spangler et $\mathrm{al}^{29}$ used a similar conjunctival allergen challenge model to that used by Friedlaender et al in $2000 .{ }^{18}$ In this prospective, doublemasked trial, 111 subjects were randomized into three groups, ie, one eye treated with azelastine and the contralateral eye treated with olopatadine, one eye treated with azelastine and the other placebo (artificial tears), and one eye treated with olopatadine and the other placebo (artificial tears). All eyes were confirmed on the first visit to have a bilateral allergic response to a known allergen, increasing the concentration until a positive reaction was elicited. On visit 2 , all eyes were challenged with the allergen to establish the intensity of their response. At a third visit, eyes were pretreated with azelastine, olopatadine, or placebo five minutes before administration of the previously determined allergen. Patients then rated the intensity of itching on a scale of $0-4$ at 30-second intervals over a total of 20 minutes. The mean itching scores of eyes treated with azelastine, olopatadine, and placebo were then compared. Although both azelastine and olopatadine controlled itching after allergen exposure significantly better than placebo, olopatadine was significantly more effective than azelastine beginning at 3.5 minutes after allergen exposure and for the duration of the 20-minute test period. Although this was a short time period, in this study olopatadine appeared to be more efficacious than azelastine for allergic conjunctivitis.

The recent major multicenter, prospective, open-label PACE (Pataday Allergic Conjunctivitis Evaluation) study compared olopatadine $0.2 \%$ once daily with azelastine $0.05 \%$ twice daily. ${ }^{1}$ Patients with allergic conjunctivitis who had recently used azelastine completed a questionnaire evaluating their previous azelastine medication $(n=49)$. All patients then replaced azelastine with olopatadine $0.2 \%$ once daily, and completed a similar survey about their experience with olopatadine seven days later. There were no differences between the two drugs in those patients who found them only somewhat effective. However, more patients (42\%-46\%) reported that olopatadine improved itching and redness more effectively than azelastine (17\%-20\%). Drop comfort, ease of use, speed of relief, and overall satisfaction were rated significantly higher by those who found olopatadine to be very effective. Although both medications decreased symptoms of allergic conjunctivitis, olopatadine was rated higher for efficacy and tolerability by patients who received the greatest symptomatic relief. Epinastine (Elestat ${ }^{\circledR}$; Inspire Pharmaceuticals, Raleigh, NC) was rated more comfortable than azelastine in other research. ${ }^{30}$

\section{Azelastine in clinical practice}

Our current ability to control allergic symptoms is greater than ever before. Our knowledge and the tools at our disposal are now substantial, including potent, multimechanism agents specifically targeting the allergic response. Isolated ocular allergy is usually very responsive to carefully selected topical medications.

More effective newer antihistamine products with multiple antiallergic mechanisms are now available. Most of these newer antihistamines can also stabilize mast cell membranes and thereby exert both indirect and direct effects on immune cells. ${ }^{8}$ The newer agents reduce application frequency and have the advantage of rapid therapeutic onset. The first such drug, olopatadine, established new records for ophthalmic pharmaceutical sales in the US, and is the standard with 
which other drugs in this class are compared. Newer drugs in this category include azelastine, epinastine, ketotifen (Zaditor ${ }^{\circledR}$; Novartis and Alway, Bausch and Lomb), bepotastine $1.5 \%$ (Bepreve $^{\circledR}$; Ista Pharmaceuticals, Irvine, CA), and nedocromil (Alocril ${ }^{\circledR}$; Allergan, Irvine, CA).

Topical multimechanism agents also enhance efficacy by inhibiting key cellular components of the allergic response. ${ }^{4}$ As potent mast cell stabilizers, they inhibit subsequent IgE/antigen-mediated release of histamine and other preformed chemical mediators that both produce acute allergic symptoms and contribute to the inflammatory response accompanying ocular allergy. ${ }^{13,8}$ Furthermore, they can also mitigate the delayed hypersensitivity reaction by inhibiting the activity of proinflammatory cells and cytokines, as well as the expression of cell surface adhesion molecules that are important in eosinophil diapedesis.

Methodic evaluation is critical in the patient with ocular redness and a chief complaint of itching to ascertain the differential diagnosis of either seasonal or perennial acute allergic conjunctivitis. Allergic conjunctivitis is a multifactorial condition with a panoply of causes, presentations, and degrees of discomfort. While these topical multimechanism agents share many properties, the percentage of patients responding to any one of these ranges from $33 \%$ to $75 \%$. Patients, who do not respond to one drug in this class, or are unable to tolerate it, may find another drug in this class to be efficacious and tolerable.

As a broad-spectrum agent, azelastine offers many of these desirable properties for management of ocular allergies. Because it can often produce maximal effect with just twice-daily dosing, azelastine is a particularly good choice for the allergic population in whom minimizing exposure to topical products and preservatives are a key concern. Even though it was rated somewhat less efficacious and may sting briefly after instillation in one out of three patients, ${ }^{4}$ many patients experienced full benefit, which they considered to be more important than the transient stinging sensation upon instillation of the azelastine eyedrop. Furthermore, with convenient administration schedules, comfort, and effectiveness, topical therapy is associated with excellent patient compliance, which is a fundamental determinant of efficacy.

Nonpharmacologic management is also an essential component in the care of patients with ocular allergy. Nonpharmacologic interventions include strategies to reduce exposure to inciting antigens, management of dry eye, and even dietary intervention. These prophylactic measures should be continued during topical therapy until allergy symptoms have abated, and to decrease the likelihood of a relapse in the continuing presence of allergens.
We cannot actually cure the allergy just yet. It is important for patients to understand this. Allergy symptom treatment may not be as glamorous or dramatic as cataract or refractive surgery or the reversal of life-threatening status asthmaticus, but favorable therapeutic results make patients happy, productive, and comfortable. By using cost-effective treatment regimens, both practical and medical, and enlisting the help of family members and other physicians, we can achieve acceptable control of most allergic symptoms. Not bad for a $40 \mu \mathrm{L}$ drop in a 6 cc eye dropper bottle.

\section{Disclosure}

The authors report no conflicts of interest in this work.

\section{References}

1. Epstein AB, van Hoven PT, Kaufman A, Carr WW. Management of allergic conjunctivitis: An evaluation of the perceived comfort and therapeutic efficacy of olopatadine $0.2 \%$ and azelastine $0.05 \%$ from two prospective studies. Clin Ophthalmol. 2009;3:329-336.

2. Bielory L, Bielory B. Ocular allergy: An allergist's perspective. Available at: http://bmctoday.net/advancedocularcare/2010/03/article. asp? $\mathrm{f}=$ ocular-allergy-an-allergists-perspective. Accessed Aug 16, 2010.

3. Pflugfelder SC. Prevalence, burden, and pharmacoeconomics of dry eye disease. Am J Manag Care. 2008;14 Suppl 3:S102-S106.

4. Bielory L, Lien KW, Bigelsen S. Efficacy and tolerability of newer antihistamines in the treatment of allergic conjunctivitis. Drugs. 2005; 65:215-218.

5. Bielory L, Buddiga P, Bigelsen S. Ocular allergy treatment comparisons: Azelastine and olopatadine. Curr Allergy Asthma Rep. 2004;4:320-325.

6. Baudouin C. Detrimental effect of preservative in eye drops: Implications for the treatment of glaucoma. Acta Ophthalmologica. 2008;86:716-726.

7. Lee JS, Lee JE, Kim N, Oum BS. Comparison of the conjunctival toxicity of topical ocular antiallergic agents. J Ocul Pharmacol Ther. 2008;24:557-562.

8. Lambiase A, Micera A, Bonini S. Multiple action agents and the eye: Do they really stabilize mast cells? Curr Opin Allergy Clin Immunol. 2009;9:454-465.

9. Casale T. The interaction of azelastine with human lung histamine $\mathrm{H}_{1}$, beta, and muscarinic receptor-binding sites. J Allergy Clin Immunol. 1989;83:771-776.

10. Hazama H, Nakajima T, Hisada T, Hamada E, Omata M, Kurachi Y. Effects of azelastine on membrane currents in tracheal smooth muscle cells isolated from the guinea-pig. Eur J Pharmacol. 1994;259: 143-150.

11. Perhach JL, Connell JT, Kemp JP. Treatment of upper and lower airway disease with azelastine. N Engl Reg Allergy Proc. 1987;8:121-124.

12. Szelenyi I, Achterrath-Tuckermann U, Schmidt J, Minker E, Paegelow I, Werner H. Azelastine: A multifaceted drug for asthma therapy. Agents Actions Suppl. 1991;34:295-311.

13. Galatowicz G, Ajayi Y, Stern ME, Calder VL. Ocular antiallergic compounds selectively inhibit human mast cell cytokines in vitro and conjunctival cell infiltration in vivo. Clin Exp Allergy. 2007;37:1648-1656.

14. Ciprandi G, Pronzato C, Passalacqua G, et al. Topical azelastine reduces eosinophil activation and intercellular adhesion molecule-1 expression on nasal epithelial cells: An antiallergic activity. JAllergy Clin Immunol. 1996;98(6 Pt 1):1088-1096.

15. Simons FE, Simons KJ. Clinical pharmacology of new histamine $\mathrm{H}_{1}$ receptor antagonist. Clin Pharmacokinet. 1999;36:329-352. 
16. Riethmuller-Winzer H, Petr G, Buker KM, Romeis P, Borbe HO. Tolerability, pharmacokinetics and dose linearity of azelastine hydrochloride in healthy subjects. Arzneimittelforschung. 1994;44:1136-1140.

17. Peter G, Romeis P, Borbe HO, Buker KM, Riethmuller-Winzen H. Tolerability and pharmacokinetics of single and multiple doses of azelastine hydrochloride in elderly volunteers. Arzneimittelforschung. 1995;45:576-581.

18. Friedlaender M, Harris J, LaVallee N, Russell H, Shilstone J. Evaluation of the onset and duration of effect of azelastine eye drops $(0.05 \%)$ versus placebo in patients with allergic conjunctivitis using an allergen challenge model. Ophthalmology. 2000;107:2152-2157.

19. Nakajima M, Nakamura S, Tokudome S, Shimada N, Yamazaki H, Yokoi T. Azelastine $N$-demethylation by cytochrome P-450 (CYP)3A4, CYP2D6, and CYP1A2 in human liver microsomes: Evaluation of approach to predict the contribution of multiple CYPS. Drug Metab Dispos. 1999;27:1381-1391.

20. Bernstein J. Azelastine hydrochloride: A review of pharmacology, pharmacokinetics, clinical efficacy and tolerability. Curr Med Res Opin. 2007;23:2441-2452.

21. Zechel HJ, Brock N, Lenke D, Achterrath-Tuckerman U. Pharmacological and toxicological properties of azelastine, a novel anti-allergic agent. Arzneimittelforschung.1981;31:1184-1193.

22. Sabbah A, Marzetto M. Azelastine eye drops in the treatment of seasonal allergic conjunctivitis or rhinoconjunctivitis in young children. Curr Med Res Opin. 1998;14:161-170.

23. Herman D, Garay R, Le Gal M. A randomized double-blind placebo controlled study of azelastine nasal spray in children with perennial rhinitis. Int J Pediatr Otorhinolaryngol. 1997;39:1-8.
24. DuBuske LM. Second-generation antihistamines: The risk of ventricular arrhythmias. Clin Ther. 1999;21:281-295.

25. Ciprandi G, Buscaglia S, Catrullo A, et al. Azelastine eye drops reduce and prevent allergic conjunctival reaction and exert anti-allergic activity. Clin Exp Allergy. 1997;27:182-191.

26. Giede-Tuch C, Westhoff M, Zarth A. Azelastine eye-drops in seasonal allergic conjunctivitis or rhinoconjunctivitis. A double-blind, randomized, placebo-controlled study. Allergy. 1998;53:857-862.

27. Nazarov O, Petzold U, Haase H, Nguyen DT, Ellers-Lenz B, Hermann R. Azelastine eye drops in the treatment of perennial allergic conjunctivitis. Arzneimittelforschung. 2003;53:167-173.

28. Canonica GW, Ciprandi G, Petzold U, Kolb C, Ellers-Lenz B, Hermann R. Topical azelastine in perennial allergic conjunctivitis. Curr Med Res Opin. 2003;19:321-329.

29. Spangler DL, Bensch G, Berdy GJ. Evaluation of the efficacy of olopatadine hydrochloride $0.1 \%$ ophthalmic solution and azelastine hydrochloride $0.05 \%$ ophthalmic solution in the conjunctival allergen challenge model. Clin Ther. 2001;23:1272-1280.

30. Torkildsen GL, Ousler GW 3rd, Gomes P. Ocular comfort and drying effects of three topical antihistamine/mast cell stabilizers in adults with allergic conjunctivitis: A randomized, double-masked crossover study. Clin Ther. 2008;30:1264-1271.
Clinical Ophthalmology

\section{Publish your work in this journal}

Clinical Ophthalmology is an international, peer-reviewed journal covering all subspecialties within ophthalmology. Key topics include: Optometry; Visual science; Pharmacology and drug therapy in eye diseases; Basic Sciences; Primary and Secondary eye care; Patient Safety and Quality of Care Improvements. This journal is indexed on

Submit your manuscript here: http://www.dovepress.com/clinical-ophthalmology-journal

\section{Dovepress}

PubMed Central and CAS, and is the official journal of The Society of Clinical Ophthalmology (SCO). The manuscript management system is completely online and includes a very quick and fair peer-review system, which is all easy to use. Visit http://www.dovepress.com/ testimonials.php to read real quotes from published authors. 